\title{
EDITORIAL
}

\section{Interferon- $\gamma$ release assays for the diagnosis of active tuberculosis: sensible or silly?}

\author{
C. Lange* ${ }^{*}$ M. Pai ${ }^{\#}$, F. Drobniewski ${ }^{\top}$ and G.B. Migliori ${ }^{+}$
}

very time Mycobacterium tuberculosis is recovered from human specimens by microbiological culture, the diagnosis of active tuberculosis (TB) is definite [1]. As culture growth of $M$. tuberculosis may take $\geqslant 2$ weeks on average, clinicians need to rely on other methods to achieve rapid diagnosis of active $\mathrm{TB}$ and justify the decision to treat the patient. The decision to initiate anti-TB treatment can be difficult, especially in children, and is currently based on different criteria including: 1) medical history and local epidemiology; 2) chest radiography; 3) the microscopic detection of acid fast bacilli (AFB); 4) amplification of $M$. tuberculosis nucleic acids from biological specimens; 5) the detection of mycobacteria-specific immune responses in the tuberculin skin test (TST); and 6) interferon (IFN)- $\gamma$ release assays (IGRAs). Furthermore, several scoring methods have been proposed to diagnose TB in children when microbiological evidence is lacking. While serological antibody-based tests have not been successful [2], novel methods for the diagnosis of TB are constantly being developed [3].

IGRAs, commercially available as the QuantiFERON-TBGold $_{\circledR}$ In Tube (QFT-GIT) test (Cellestis, Carnegie, Australia) and as the T-SPOT.TB ${ }_{\circledR}$ test (Oxford Immunotec, Abingdon, $\mathrm{UK})$, are now established in many countries as advanced tools for the immunodiagnosis of latent TB infection (LTBI). In contrast to the mixture of antigens in the purified protein derivate, which is used in the in vivo TST, the IGRA detects effector memory responses to a limited number of specific RD1 peptides by peripheral blood mononuclear cells (PBMC) ex vivo. The genes that encode for the RD1 proteins are absent in the bacille Calmette-Guérin (BCG) vaccine strain. Consequently, in BCG-vaccinated recent contacts of infectious cases, IGRA responses correlate better to $M$. tuberculosis exposure than the TST [4-8]. Therefore, it is plausible that IGRAs are superior to the TST in predicting the development of active TB in BCG-vaccinated recent contacts. A prospective

\footnotetext{
*Division of Clinical Infections, Research Center Borstel, Borstel, Germany. " Dept of Epidemiology and Biostatistics, McGill University, Montreal, QC, Canada. "Institute for Cell and Molecular Sciences, Barts and the London School of Medicine and Dentistry, Queen Mary College, London, UK. ${ }^{+}$WHO Collaborating Centre for TB and Lung Diseases, Fondazione S. Maugeri, Care and Research Institute, Tradate, Italy.
}

STATEMENT OF INTEREST: Statements of interest for $\mathrm{C}$. Lange and F. Drobniewski can be found at www.erj.ersjournals.com/misc/statements.dtl

CORRESPONDENCE: G.B. Migliori, WHO Collaborating Centre for TB and Lung Diseases, Fondazione S. Maugeri, Care and Research Institute, via Roncaccio 16, 21049, Tradate, Italy. Fax: 39 0331829402. E-mail: giovannibattista.migliori@fsm.it study that evaluated the predictive value of IGRA for the development of active TB in contacts of patients with $\mathrm{TB}$ suggested superiority over the TST [9], although the effect was less pronounced in another study in children [10], and no significant difference in predictive values was found in an investigation with an in-house IGRA in the Gambia [11].

The role of IGRA for the diagnosis of active TB is less clear, particularly in children. In $10-15 \%$ of children [12] and $\sim 50 \%$ of adult cases [13] with active pulmonary TB (those with a positive sputum AFB smear), immunodiagnosis by TST or IGRA is unnecessary and makes little sense. In this situation, molecular nucleic acid amplification tests (NAAT) can be applied rapidly to identify the bacilli as $M$. tuberculosis (in contrast to nontuberculous mycobacteria) and provide useful information on the presence of rifampicin resistance [14]. Immunodiagnosis may have some utility when AFB-sputum smears, and when available NAAT, of TB suspects are negative. In those with negative sputum smears, a common finding in children, positive IGRA results (when performed on PBMC) and/or TST results are of limited direct value as immunodiagnostic tests are not likely to distinguish active TB from LTBI.

In the current issue of the European Respiratory Journal, KAMPMANN et al. [15] extend the known inability of IGRAs to distinguish between active TB and LTBI in adults to children. A cohort of 209 children from the UK were investigated by the two commercially available IGRA tests, in parallel with the TST, for the presumptive diagnosis of LTBI $(n=118)$ or active TB $(n=91)$. Neither the presence of a positive IGRA result nor the magnitude of the assayed immune response (IFN- $\gamma$ $\left(\mathrm{IU} \cdot \mathrm{mL}^{-1}\right)$ in the ELISA or number of spot forming cells per 250,000 PBMC in the ELISPOT) was able to discriminate between active TB and LTBI.

\section{WHY IS IT THAT IGRA CANNOT DISTINGUISH BETWEEN ACTIVE TB AND LTBI?}

The answer probably lies in the limitations of using peripheral blood for the assays. IGRAs have been designed to measure the IFN- $\gamma$ production by peripheral blood cells, predominantly a distinct subgroup of T-lymphocytes. However, only $2-5 \%$ of the lymphocyte pool of the human body circulates in the blood [16]. The number of effector memory T-cells (the population of cells likely to be the predominant source of the early production of IFN- $\gamma$ following ex vivo antigen contact in IGRA) are very low in the blood. In active TB, the memory Tcells that develop into effector memory T-cells are recruited to the site of infection [17]. Thus, effector memory T-cell 
responses assayed on PBMC may only provide background information about the activation of the immune system in active disease. Consequently, IGRA responses assayed in mononuclear cells from local infected compartments should provide better discrimination of active TB from LTBI than responses with PBMC alone. Indeed, $M$. tuberculosis specific Tcells have been found in both immunocompetent and immunosuppressed individuals to be expanded among lymphocytes from the bronchoalveolar lavage (BAL), pleural effusion, ascites, pericardial effusion and cerebrospinal fluid in patients with AFB-sputum smear negative pulmonary, pleural, peritoneal, pericardial and meningeal TB [18-26]. However, the literature describes studies with relatively small numbers of patients or single case reports. Larger prospective clinical trials are needed to evaluate $M$. tuberculosis specific BAL-IGRA in order to distinguish between active TB and LTBI in suspects of pulmonary TB with negative AFB-sputum smears.

\section{DO IGRAS HAVE A ROLE IN THE DIAGNOSIS OF ACTIVE TB?}

Immune-based tests, such as IGRA and TST, do not directly detect $M$. tuberculosis but merely indicate a cellular immune response to recent or remote sensitisation with $M$. tuberculosis [27]. As IGRA cannot distinguish between LTBI and active TB, a positive IGRA result may not necessarily indicate active TB (this is almost certainly true in high TB prevalence settings). Furthermore, an isolated negative IGRA result would not conclusively rule out active disease in an individual suspected to have TB; this also applies to the TST. This is because IGRA, like the TST, do not detect all cases of culture-confirmed active TB; meta-analyses suggest that IGRA have a sensitivity of $\sim 70$ $90 \%$ in active TB (with ELISPOT showing higher sensitivity than ELISA-based formats), and sensitivity may be lower in high TB incidence settings [28, 29]. However, meta-analysis estimates of sensitivity are mainly based on studies in adults. KAMPMANN et al. [15] found the TST to have a sensitivity of $83 \%$ in active TB, compared with $80 \%$ for QFT-GIT and $58 \%$ for TSPOT.TB. While the TST and QFT-GIT sensitivity estimates are consistent with previous meta-analyses estimates, the reported sensitivity of T-SPOT.TB (58\%) is substantially lower than prior estimates. Technical errors and specimen handling issues should always be kept in mind but these assays have been performed with appropriate controls and one must also consider that there may be underlying immunophysiological reasons for the lower T-SPOT.TB sensitivity in children as suggested by KAMPMANN et al. [15].

Regardless, the study by KAMPMANN et al. [15] further confirms the need to be cautious in ruling out active TB on the basis of an isolated negative IGRA result. However, would the situation improve if both TST and IGRA were used in combination? The negative predictive value for $M$. tuberculosis infection is $>95 \%$ if both IGRA and TST test results are negative [30, 31]. Therefore, negative combined test results almost exclude the diagnosis of both LTBI and active TB in immunocompetent individuals. KAMPMANN et al. [15] have now extended our knowledge about this important finding to children being evaluated for active TB. As with any "negative result" it is essential that appropriate controls are in place to detect deleterious handling of the samples that may increase the possibility of false negative results.
As previously suggested, for the diagnosis of active TB, positive test results of IGRA and TST will be most useful where the pre-test probability of active TB is high and the pretest probability of latent infection is low. This applies best to persons with clinical manifestations of active TB who lack risk factors of exposure to M. tuberculosis [32]. Certainly, this is not a situation that applies to individuals from countries with a high incidence of TB.

\section{WHAT IS THE CURRENT EVIDENCE FOR USE OF IGRAS IN CLINICAL PRACTICE?}

There may be two situations where IGRAs offer additional clinical value beyond the TST: in those who are vaccinated with BCG after infancy or in those receive repeated BCG boosters. IGRAs are superior to the TST because they are unlikely to be falsely positive. The immune memory identified by IGRAs probably better correlates with the risk of developing active TB, although the effect may not be so convincing in children and in individuals not vaccinated with BCG. For the diagnosis of active TB in non-immunocompromised hosts, a combined negative test result in both IGRA and TST may be used as a rule-out test of very high specificity. However, for adults, contrary to the situation in children, there are effective rapid molecular diagnostics for $M$. tuberculosis detection and screening of multi-drug resistance, and waiting to read the TST to complement the IGRA may be a much less effective strategy in reality.

Our knowledge on the positive and negative predictive values of IGRA for the development of active TB in the most vulnerable populations with immunosuppression (e.g. with HIV infection, chronic renal failure, solid organ or stem cell transplantation and in those scheduled for anti-tumour necrosis factors therapies) is still very limited. Results from cross sectional cohort studies [33-35] suggest that frequencies of positive immune responses to IGRA are higher in severely immunosuppressed individuals than to the TST, but data from longitudinal studies on the incidence of TB in those who were tested are, unfortunately, still missing. Finally, it is still unclear if IGRA offer better predictive value in high TB incidence countries.

In conclusion, to date, none of the immune-based tests (TST, IGRA from peripheral blood or antibody-based serological tests) can replace conventional tests such as smear microscopy, culture and NAAT for the diagnosis of active TB. In contrast, local immunodiagnosis by BAL-ELISPOT [36] or fluorescenceactivated cell sorter (FACS) analysis [23] is a promising method under evaluation to distinguish LTBI from active TB in suspects of pulmonary $\mathrm{TB}$ with negative AFB-sputum smears and NAAT results. This approach may be most applicable for a rapid decision to initiate anti-TB treatment in settings where bronchoscopy is routinely performed in patients with negative AFB smears and where the technology for ELISPOT and/or FACS analysis exists. However, species identification and drug resistant testing is currently not possible by immune-based tests.

Until accurate immunological biomarkers are identified, the gold standard for the diagnosis of active tuberculosis continues to be the direct detection, isolation or amplification of Mycobacterium tuberculosis from clinical specimens. 


\section{REFERENCES}

1 Migliori GB, Raviglione MC, Schaberg T, et al. Tuberculosis management in Europe. Task Force of the European Respiratory Society (ERS), the World Health Organisation (WHO) and the International Union against Tuberculosis and Lung Disease (IUATLD) Europe Region. Eur Respir J 1999; 14: 978-992.

2 Steingart KR, Dendukuri N, Henry M, et al. Performance of purified antigens for serodiagnosis of pulmonary tuberculosis: a meta-analysis. Clin Vaccine Immunol 2009; 16: 260-276.

3 Pai M, O'Brien R. New diagnostics for latent and active tuberculosis: state of the art and future prospects. Semin Respir Crit Care Med 2008; 29: 560-568.

4 Diel R, Nienhaus A, Lange C, et al. Tuberculosis contact investigation with a new, specific blood test in a lowincidence population containing a high proportion of BCGvaccinated persons. Respir Res 2006; 7: 77.

5 Ewer K, Deeks J, Alvarez L, et al. Comparison of T-cellbased assay with tuberculin skin test for diagnosis of Mycobacterium tuberculosis infection in a school tuberculosis outbreak. Lancet 2003; 361: 1168-1173.

6 Kang YA, Lee HW, Yoon HI, et al. Discrepancy between the tuberculin skin test and the whole-blood interferon gamma assay for the diagnosis of latent tuberculosis infection in an intermediate tuberculosis-burden country. JAMA 2005; 293: 2756-2761.

7 Diel R, Ernst M, Döscher G, et al. Avoiding the effect of BCG-vaccination in detecting Mycobacterium tuberculosis infection with a blood test. Eur Respir J 2006; 28: 16-23.

8 Drobniewski F, Balabanova Y, Zakamova E, et al. Rates of latent tuberculosis in health care staff in Russia. PLoS Med 2007; 4: e55.

9 Diel R, Loddenkemper R, Meywald-Walter K, et al. Predictive value of a whole blood IFN- $\gamma$ assay for the development of active tuberculosis disease after recent infection with Mycobacterium tuberculosis. Am J Respir Crit Care Med 2008; 177: 1164-1170.

10 Bakir M, Millington KA, Soysal A, et al. Prognostic value of a T-cell-based, interferon- $\gamma$ biomarker in children with tuberculosis contact. Ann Intern Med 2008; 149: 777-787.

11 Hill PC, Jackson-Sillah DJ, Fox A, et al. Incidence of tuberculosis and the predictive value of ELISPOT and Mantoux tests in Gambian case contacts. PLOS ONE 2008; 3: e1379.

12 Newton SM, Brent AJ, Anderson S, et al. Paediatric tuberculosis. Lancet Infect Dis 2008; 8: 498-510.

13 Daniel TM. The rapid diagnosis of tuberculosis: a selective review. J Lab Clin Med 1990; 116: 277-282.

14 Sam IC, Drobniewski F, More P, et al. Mycobacterium tuberculosis and rifampin resistance, United Kingdom. Emerg Infect Dis 2006; 12: 752-759.

15 Kampmann B, Whittaker E, Williams A, et al. Interferon- $\gamma$ release assays do not identify more children with active tuberculosis than the tuberculin skin test. Eur Respir J 2009; 33: 1371-1379.

16 Westermann J, Pabst R. Distribution of lymphocyte subsets and natural killer cells in the human body. Clin Investig 1992; 70: 539-544.

17 Jafari C, Lange C. Suttons's Law: local immunodiagnosis of tuberculosis. Infection 2008; 36: 510-514.
18 Jafari C, Ernst M, Kalsdorf B, et al. Rapid diagnosis of smear-negative tuberculosis by bronchoalveolar lavage enzyme-linked immunospot. Am J Respir Crit Care Med 2006; 174: 1048-1054.

19 Jafari C, Ernst M, Strassburg A, et al. Local immunodiagnosis of pulmonary tuberculosis by enzyme-linked immunospot. Eur Respir J 2008; 31: 261-265.

20 Losi M, Bossink A, Codecasa L, et al. Use of a T-cell interferon- $\gamma$ release assay for the diagnosis of tuberculous pleurisy. Eur Respir J 2007; 30: 1173-1179.

21 Wilkinson KA, Wilkinson RJ, Pathan A, et al. Ex vivo characterization of early secretory antigenic target 6specific T-cells at sites of active disease in pleural tuberculosis. Clin Infect Dis 2005; 40: 184-187.

22 Lange C, Hellmich B, Ernst M, et al. Rapid immunodiagnosis of tuberculosis in a woman receiving anti-TNF therapy. Nat Clin Pract Rheumatol 2007; 3: 528-534.

23 Strassburg A, Jafari C, Ernst M, et al. Rapid diagnosis of pulmonary TB by BAL enzyme-linked immunospot assay in an immunocompromised host. Eur Respir J 2008; 31: 1132-1135.

24 Thomas MM, Hinks TS, Raghuraman S, et al. Rapid diagnosis of Mycobacterium tuberculosis meningitis by enumeration of cerebrospinal fluid antigen-specific T-cells. Int J Tuberc Lung Dis 2008; 12: 651-657.

25 Köesters K, Nau R, Bossink A, et al. Rapid diagnosis of CNS tuberculosis by a T-cell interferon- $\gamma$ release assay on cerebrospinal fluid mononuclear cells. Infection 2008; 36: 597-600.

26 Nemeth J, Winkler HM, Zwick RH, et al. Recruitment of Mycobacterium tuberculosis specific CD4+T cells to the site of infection for diagnosis of active tuberculosis. J Intern Med 2009; 265: 163-168.

27 Mack U, Migliori GB, Sester M, et al. LTBI: latent tuberculosis infection or lasting immune responses to Mycobacterium tuberculosis? A TBNET consensus statement. Eur Respir J 2009; 33: 956-973.

28 Menzies D, Pai M, Comstock G. Meta-analysis: new tests for the diagnosis of latent tuberculosis infection: areas of uncertainty and recommendations for research. Ann Intern Med 2007; 146: 340-354.

29 Pai M, Zwerling A, Menzies D. Systematic review: T-cellbased assays for the diagnosis of latent tuberculosis infection: an update. Ann Intern Med 2008; 149: 177184.

30 Dosanjh DP, Hinks TS, Innes JA, et al. Improved diagnostic evaluation of suspected tuberculosis. Ann Intern Med 2008; 148: 325-336.

31 Goletti D, Stefania C, Butera O, et al. Accuracy of immunodiagnostic tests for active tuberculosis using single and combined results: a multicenter TBNET-Study. PLoS ONE 2008; 3: e3417.

32 Menzies D. Using tests for latent tuberculous infection to diagnose active tuberculosis: can we eat our cake and have it too? Ann Intern Med 2008; 148: 398-399.

33 Stephan C, Wolf $\mathrm{T}$, Goetsch U, et al. Comparing QuantiFERON-tuberculosis gold, T-SPOT tuberculosis and tuberculin skin test in HIV-infected individuals from a low prevalence tuberculosis country. AIDS 2008; 22: 2471-2479. 
34 Rangaka MX, Wilkinson KA, Seldon R, et al. Effect of HIV-1 infection on T-cell-based and skin test detection of tuberculosis infection. Am J Respir Crit Care Med 2007; 175: 514-520.

35 Ponce de Leon D, Acevedo-Vasquez E, Alvizuri S, et al. Comparison of an interferon-gamma assay with tuberculin skin testing for detection of tuberculosis (TB) infection in patients with rheumatoid arthritis in a TB-endemic population. J Rheumatol 2008; 35: 776-781.

36 Jafari C, Thijsen S, Bossink A, et al. Comparison of BAL ELISPOT and NAT for the diagnosis of smear-negative tuberculosis-a TBNET study. Eur Respir J 2008; 32: Suppl. $52,1 \mathrm{~s}$. 